\title{
Advance Care Planning in A Preoperative Clinic: A Retrospective Chart Review
}

\author{
Shilpee Sinha, MD, FACP ${ }^{1,2,3,4,5}$, Rachel N. Gruber, MS ${ }^{1,6,7}$, Ann H. Cottingham, MAR, MA ${ }^{7,3,6,7}$, \\ Barb Nation, RN, CHPN ${ }^{2,3}$, Kathleen A. Lane, $\mathrm{MS}^{3,6,8}, \mathrm{Na} B \mathrm{BO}, \mathrm{MSPH}^{3,6,8}$, and Alexia Torke, \\ $M D, M S^{2,5,6,9,10,11}$
}

\begin{abstract}
'Advanced Scholars Program for Internists in Research and Education (ASPIRE), Indiana University (IU) School of Medicine, Indianapolis, IN, USA; ${ }^{2}$, IU Health Physicians, Indianapolis, IN, USA; ${ }^{3}$, Indiana University School of Medicine, Indianapolis, IN, USA; ${ }^{4}$ IUH Department of Palliative Care, Academic Health Center, Indiana University School of Medicine, Indianapolis, IN, USA; ${ }^{5}$ Division of General Internal Medicine and Geriatrics, School of Medicine, Indiana University, Indianapolis, IN, USA; ${ }^{6} V A$ Health Services Research and Development Center for Health Information and Communication, Richard L. Roudebush VA Medical Center, Indianapolis, IN, USA; ${ }^{7}$ Indiana University Center for Health Services and Outcomes Research, Regenstrief Institute, Inc., Indianapolis, IN, USA; ${ }^{8}$ Department of Biostatistics, Indiana University School of Medicine, Indianapolis, IN, USA; ${ }^{9}$ Indiana University Center for Aging Research, Regenstrief Institute, Inc., Indianapolis, IN, USA; ${ }^{10}$ Fairbanks Center for Medical Ethics, IU Health, Indianapolis, IN, USA; "'Daniel F. Evans Center for Spiritual and Religious Values in Healthcare, IU Health, Indianapolis, IN, USA.
\end{abstract}

$\mathrm{J}$ Gen Intern Med 34(4):523-5

DOI: $10.1007 / \mathrm{s} 11606-018-4744-8$

(c) Society of General Internal Medicine 2019

\section{INTRODUCTION}

Patients seen in preoperative testing clinics are at an increased risk of surgical complications and most are incapacitated for during anesthesia. Advance directives (ADs) are important to guide care in the event of emergencies when patients are unable to speak for themselves. The goal of this study was to determine the frequency with which ADs are completed for patients seen in preoperative clinics prior to elective surgery and identify demographic and clinical characteristics associated with having ADs available in the electronic medical record (EMR).

\section{METHODS}

\section{Study Design}

A retrospective EMR chart review was conducted on 400 consecutive patients who underwent a preoperative evaluation in a preoperative clinic from February 1 through early March 2017 at two high-volume hospitals in a midwestern urban academic tertiary referral center.

\section{Data Collection}

All raters first completed chart reviews of the same 30 patients to calculate interrater reliability.

We collected demographic variables, elevated perioperative risk of surgery, ${ }^{1}$ Revised Cardiac Risk Index (RCRI) $>1 \%,{ }^{2}$ and presence of revised Charleston comorbid conditions. ${ }^{3}$ Functional capacity was reported in the EMR as either metabolic equivalents (METS) scale (1 through 4) or as "good," "borderline," or "poor." We dichotomized

Published online January 2, 2019 patients' functional capacity as "good" or 4 METS versus and "other" or 1-3 METS.

Reviewers assessed the presence of AD documents scanned into the EMR, including an SDM, a living will, or either (any AD). The study was approved by the Indiana University Institutional Review Board.

\section{Statistical Analysis}

Patient characteristics were compared based upon the presence of having either type of AD documents in the EMR using two sample $t$ tests for the continuous variables and Fisher's exact tests for the categorical variables. All preoperative patient clinical and demographic variables significant in the univariate model at the alpha $=0.15$ level were included in multiple logistic regression modeling of the $\mathrm{AD}$ outcome. Both stepwise and backward methods were used to identify a final parsimonious model with all variables significant at the alpha $=0.05$ level.

\section{RESULTS}

Interrater reliability for the Charlson ${ }^{3}$ score showed interclass correlations from $0.76-0.97$. The range for Cohen's kappa coefficients on categorical data ranged from 0.37 to 1 , and percentage agreement ranged from 67 to $100 \%$ for pairwise comparisons.

We found 64 (16.0\%) of patients had evidence of either $\mathrm{AD}$ scanned into the EMR, $43(10.8 \%)$ had an SDM, and 46 $(11.5 \%)$ had a living will. In a bivariate analysis, age 54.7 vs. $31.3 \%, p=0.0005)$ and higher functional status (17.7 vs. $7.0 \%, p=0.0118$ ) were associated with the presence of any AD. There were no differences between those with and without ADs with complications or death (Table 1).

Multiple logistic regression examining preoperative characteristics associated with any $\mathrm{AD}$ found patients age 65 and older (odds ratio (OR), 2.85; 95\% confidence interval (CI), 
Table 1 Comparison of Patient Characteristics and Hospital Events by Presence of any Advance Directive (Surrogate Decision Maker or Living Will) in the Electronic Medical Record

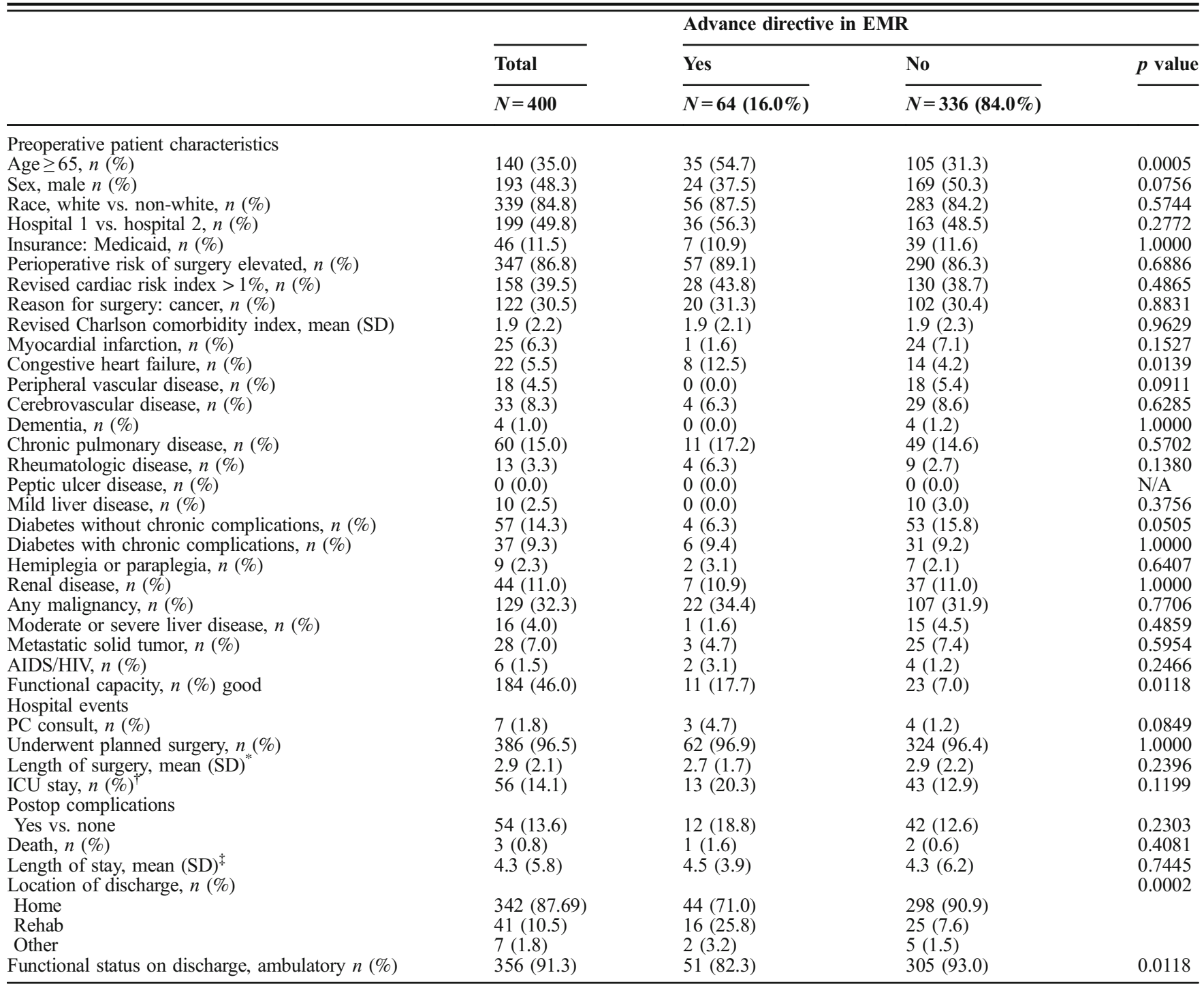

*Length of surgery was calculated in hours from anesthesia records

${ }^{t}$ Patients who required any length of ICU stay

${ }^{*}$ Length of stay was days spent in hospital

$1.63,4.98)$ and those with congestive heart failure (OR, 4.04; $95 \%$ CI, 1.55, 10.54) had significantly higher odds of having ADs. Men had significantly lower odds of having AD than females (OR, 0.56; 95\% CI, $(0.32,0.99)$.

There were $14(3.5 \% ; n=10 \mathrm{SDM} / 8$ living will) patients who had not reported having an $\mathrm{AD}$ at the clinic visit but had one documented in the EMR prior to surgery and 66 (16.5\%) who reported having an $\mathrm{AD}$ at the clinic visit but did not have one documented in the EMR prior to surgery (Table 2).

\section{DISCUSSION}

Patients undergoing elective surgery and evaluated in a PAT had a low prevalence of AD, a finding similar to reports that identify low levels of ACP completion in other settings, ${ }^{1,4}$ and studies finding that the integration of ACP conversations into the preoperative clinic process is not an established norm. ${ }^{5}$ Lack of AD documentation places a burden on family members to make decisions on

Table 2 Frequency of Advance Directive (AD) Reported to Exist by Patient Compared to Scanned to Electronic Medical Record (EMR), $n(\%)$

\begin{tabular}{lllll}
\hline \hline $\begin{array}{l}\text { AD reported to exist } \\
\text { by patient }\end{array}$ & & \multicolumn{2}{l}{ AD scanned to EMR } & \\
\cline { 5 - 5 } & & Yes & No & Total \\
\hline Yes & $50(12.5)$ & $66(16.5)$ & $116(29.0)$ \\
No & $14(3.5)$ & $270(67.5)$ & $284(71.0)$ \\
Total & $64(16.0)$ & $336(84.0)$ & $400(100.0)$ \\
\hline
\end{tabular}


their loved one's behalf without patient guidance and can lead to care that does not comport with the patient's preference. Also, some ADs that patients report completing are not available in the EMR where clinicians could access them when needed. A limitation of this study was that it included only one health system. Local practice patterns may differ at other hospitals. Our findings suggest that there is a significant opportunity for improvement in ACP in this clinical setting. Future research should focus on processes to make ACP more available to high-risk patient populations. $^{6}$

Acknowledgements: The authors would like to acknowledge Greg Sachs and Richard M. Frankel for their mentorship through the IUSM ASPIRE program.

Corresponding Author: Shilpee Sinha, MD, FACP; IUH Department of Palliative Care, Academic Health Center, Indiana University School of Medicine, 1633 N. Capitol Ave., MT 301, Indianapolis, IN 46202, USA (e-mail: ssinha@iuhealth.org).

Funding Dr. Torke was supported by a Midcareer Investigator Award in Patient-Oriented Research (K24 AG053794).

\section{Compliance with Ethical Standards:}

Conflict of Interest: The authors declare that they do not have a conflict of interest.

Publisher's Note: Springer Nature remains neutral with regard to jurisdictional claims in published maps and institutional affiliations.

\section{REFERENCES}

1. Rao JK, Anderson LA, Lin FC, Laux JP. Completion of advance directives among U.S. consumers. Am J Prev Med 2014;46(1):65-70.

2. Lee TH, Marcantonio ER, Mangione CM, et al. Derivation and prospective validation of a simple index for prediction of cardiac risk of major noncardiac surgery. Circulation 1999;100(10):1043-1049.

3. Charlson ME, Pompei P, Ales KL, MacKenzie CR. A new method of classifying prognostic comorbidity in longitudinal studies: development and validation. J Chron Dis 1987;40(5):373-383.

4. Lovell A, Yates P. Advance Care Planning in palliative care: a systematic literature review of the contextual factors influencing its uptake 20082012. Palliat Med 2014;28(8):1026-1035.

5. Grimaldo DA, Wiener-Kronish JP, Jurson T, Shaughnessy TE, Curtis JR, Liu LL. A randomized, controlled trial of advanced care planning discussions during preoperative evaluations. Anesthesiology 2001;95(1):43-50; discussion 45A.

6. Ernst KF, Hall DE, Schmid KK, et al. Surgical palliative care consultations over time in relationship to systemwide frailty screening. JAMA Surg 2014;149(11):1121-1126. 